\title{
Observation of out-of-plane unidirectional anisotropy in MgO-capped planar nanowire arrays of $\mathrm{Fe}$
}

\author{
S. K. Arora, ${ }^{1, a)}$ B. J. O'Dowd, ${ }^{1}$ D. M. Polishchuk, ${ }^{2}$ A. I. Tovstolytkin, ${ }^{2}$ P. Thakur, ${ }^{3}$ \\ N. B. Brookes, ${ }^{3}$ B. Ballesteros, ${ }^{4}$ P. Gambardella, ${ }^{4}$ and I. V. Shvets ${ }^{1}$ \\ ${ }^{1}$ Centre for Research on Adaptive Nanostructures and Nanodevices (CRANN) and School of Physics, \\ Trinity College Dublin, Dublin 2, Ireland \\ ${ }^{2}$ Department of Thin Films Physics, Institute of Magnetism, National Academy of Sciences of Ukraine, \\ Vernadsky Blvd., 36b, Kyiv 03142, Ukraine \\ ${ }^{3}$ European Synchrotron Radiation Facility, BP220, 38043 Grenoble Cedex, France \\ ${ }^{4}$ Catalan Institute of Nanotechnology (ICN-CIN2), UAB Campus, E-08193 Barcelona, Spain
}

(Received 2 July 2013; accepted 12 September 2013; published online 1 October 2013)

\begin{abstract}
We report on the effect of cap layer material on the magnetic properties and aging of the Fe-NW (nanowire) arrays grown on oxidized vicinal Si (111) templates using atomic terrace low angle shadowing technique. We find that the Fe-NW arrays capped with metallic (Ag) layers do not show any sign of degradation with aging, whereas NW arrays capped with insulating dielectric $(\mathrm{MgO})$ layers show degradation of the saturation magnetization and an out-of-plane unidirectional anisotropy. We find that this out-of-plane unidirectional anisotropy competes with the shape anisotropy which is still the dominant anisotropy. The origin of this additional anisotropy is explained on the basis of oxidation of Fe due to the presence of $\mathrm{MgO}$ that leads to the formation of an oxide interlayer. This oxide interlayer forms at the expense of NW materials, leading to reduction in the thickness of some of the Fe-NWs within the array, and orients their magnetic moments out-of-plane. The reduction in NW thickness and the presence of Fe-O interlayer facilitates stabilization of this anisotropy. Our model is supported by X-ray absorption spectroscopy studies performed as a function of aging, which suggests that the oxide interlayer thickness increases with aging. (C) 2013 AIP Publishing LLC. [http://dx.doi.org/10.1063/1.4823514]
\end{abstract}

\section{INTRODUCTION}

Unidirectional anisotropy (also known as exchange bias) in magnetically coupled bilayer system of ferromagnetic (FM) and antiferromagnetic (AFM) materials has been a topic of great research interest owing to the controversy related to the possible mechanisms which govern this effect and its application potential in spin electronic devices, e.g., magnetic memory and sensing applications. ${ }^{1-5}$ In understanding the origin of this unidirectional anisotropy, determining the magnetic properties of the AFM layer and FM-AFM interface is crucial. This is a challenging task as the volume of the material at the interface is small, and probing magnetic property of an AFM material is not simple due to their vanishingly small magnetic moments. However, recent advances in the $\mathrm{x}$-ray and neutron scattering techniques have enabled exploration of the magnetic response of interfaces and improved understanding of the phenomena. Our understanding of the domain states and the behavior of interface magnetism has advanced considerably using these new techniques, allowing us to better analyze the nature of the exchange bias mechanism. ${ }^{6-8}$

Most studies devoted to understanding the mechanism of exchange bias have been performed in systems with in-plane anisotropy of the FM. ${ }^{1-8}$ For applications, however, systems with perpendicular unidirectional anisotropy are more

\footnotetext{
a) Author to whom correspondence should be addressed. Electronic mail: aroras@tcd.ie
}

desirable due to the advantage of greater thermal stability of spin transfer torque devices. Recently, perpendicular exchange bias has been demonstrated in $\mathrm{CoFeB} / \mathrm{MgO}$ bilayer, $\mathrm{Pt} / \mathrm{Co}$ multilater, SmGdAl/SmAl bilayers, and DyCo/Ta/FeGd spin valve structures. ${ }^{9-11}$

The main indication of the existence of unidirectional anisotropy is the shift of the hysteresis loop along the field axis after field cooling from above the Neel temperature, $T_{\mathrm{N}}$, of the AFM (and below the Curie temperature, $T_{\mathrm{C}}$, of the FM) in materials characterized by presence of FM-AFM interfaces. ${ }^{1}$ An increase of the coercivity, $H_{\mathrm{C}}$, is also observed below $T_{\mathrm{N}}$. The presence of exchange bias or unidirectional anisotropy, however, can also be detected by performing ferromagnetic resonance (FMR) measurement of the effective anisotropy field. ${ }^{1-4}$ In FM films the anisotropy field is symmetric with respect to $180^{\circ}$ rotations of the magnetization. Exchange bias breaks this symmetry, resulting in a unidirectional anisotropy field. From the resonance position and line shape information about the exchange bias and anisotropies can be obtained. Exchange coupled structures exhibit the unidirectional anisotropy with a $K_{\mathrm{ud}} \cos \theta$ angular dependence of the magnetic torque, rather than $K_{\text {ua }} \sin 2 \theta$, as the common uniaxial anisotropy, where $\theta$ is the angle between the magnetization and the anisotropy axis and $K_{\mathrm{ud}}$ and $K_{\text {ua }}$ are the unidirectional and FM uniaxial anisotropy constants, respectively.

Recent advances in nanofabrication techniques have propelled a renewed interest in nanostructures in general and exchange biased ones in particular. It is well known that the 
magnetic properties (e.g., coercivity or remanence) of low dimensional magnetic structures depend strongly on their size, aspect ratio, or shape. ${ }^{1,12-14}$ Such effects could become even more complex in exchange biased nanostructures. There are very few reports that address the effects of miniaturization on the exchange bias properties of such systems. ${ }^{12,15-18}$ Exchange bias has been studied in FM-AFM wires with average widths of 100-400 nm, and the results seem to be contradictory for the wires having different composition but the same structure type. ${ }^{15-21}$ An increase in the exchange bias field $\left(H_{\mathrm{E}}\right)$ was observed $\left(H_{\mathrm{E}} \propto 1 / w\right)$ as the lateral size was reduced from continuous films to submicron dimensions, ${ }^{15,16}$ while Fraune $e t a l .{ }^{18}$ reported that $H_{\mathrm{E}}$ is insensitive to the wire width $w$. Some studies (see, for example, Refs. 19 and 20) even reported decrease in $H_{\mathrm{E}}$ with $w\left(H_{\mathrm{E}} \propto w\right)$.

One of the important material systems is Fe-MgO based bilayer and related structures, whose magnetic and spin transport properties are intensely investigated. ${ }^{22-26}$ Magnetic and electronic properties of these heterostructures are affected by the nature of interface, which depends upon the nature of defects and disorder in constituent layers, and to the extent of intermixing at the $\mathrm{Fe}-\mathrm{MgO}$ interface. Oh et $_{\mathrm{al}}{ }^{22}$ note that the formation of $\mathrm{FeO}$ cannot be avoided and suggest that $\mathrm{FeO}$ and $\mathrm{MgO}$ coexist at the interface in an entropically stabilized phase. It has also been found that the non-collinear spin structure of the $\mathrm{Fe}-\mathrm{MgO}$ interface facilitates in stabilization of perpendicular magnetic anisotropy in ultrathin structures. ${ }^{26}$ Most of previous studies in this material system were focused on 2-D bilayer system and did not address the influence of reduced dimensions, i.e., the case of nanowires (NWs).

In this report, we present a systematic study on the evolution of unidirectional anisotropy in planar Fe-NW arrays and role of the cap layer material in determining its extent. We further show that for $\mathrm{MgO}$ capped $\mathrm{Fe}-\mathrm{NWs}$, this unidirectional anisotropy is related to the presence of an interfacial oxide layer whose thickness increases at the expense of NW's material with aging. The effect becomes more pronounced as the aging progresses and leads to an enhanced unidirectional anisotropy. Our conclusions are further supported by the x-ray absorption spectroscopy measurements which exhibit an enhanced Fe-3d and O-2p hybridization.

\section{EXPERIMENTAL}

Planar NW arrays of Fe (25 nm average wire width) used in the present study were fabricated on highly regular step-bunched templates of oxidized vicinal $\mathrm{Si}$ (111) by employing a shallow angle deposition technique named ATLAS (atomic terrace low angle shadowing). Two sets of Fe-NW arrays were grown under identical conditions at room temperature by depositing $\mathrm{Fe}$ at an angle of $3^{\circ}$ in an ascending step direction. The templates used in the present study were highly regular step-bunched surfaces of vicinal Si with $140 \mathrm{~nm}$ average periodicity (miscut of $3^{\circ}$ along the $\langle 11-2\rangle$ crystallographic direction). Details of the template preparation method and ATLAS deposition technique are given elsewhere. ${ }^{12,27}$

The two sets of samples used for FMR studies differ from one another only by the cap layer material. For Set 1 ,
NWs were capped with $10 \mathrm{~nm} \mathrm{Ag}$ layer. For Set 2, a $20 \mathrm{~nm}$ $\mathrm{MgO}$ cap layer was deposited on top of the NW array. The cap layers were deposited at normal incidence angle for all samples. The average width and thickness of the Fe-NWs were determined from the analysis of atomic force microscopy scans taken over various locations on the NW array samples deposited without a capping layer. The average width and thickness of Fe NWs, as well as the sample designations used in this work, are shown in Table I.

FMR studies were carried out at room temperature with the use of an X-band ELEXSYS E500 spectrometer equipped with an automatic goniometer. The operating frequency was $\nu=9.44 \mathrm{GHz}$ (X-band). For the FMR investigation the samples were cut into samples of smaller size with dimensions of $3 \times 3 \times 0.5 \mathrm{~mm}^{3}$. The geometry of FMR measurements relative to a Fe NWs array is shown in Fig. 1(a). The zero value of theta $\left(\theta_{h}=0^{\circ}\right)$ was set to the angle where the resonance field $H_{\text {res }}$ reached maximum. The angular dependences were studied in the range of $\theta_{h}$ from $0^{\circ}$ to $360^{\circ}$.

Element specific $\mathrm{x}$-ray absorption (XAS) and x-ray magnetic circular dichroism (XMCD) experiments were carried out in total electron yield (TEY) mode at the ID08 beam-line of the European Synchrotron Radiation Facility, ESRF. To study the depth dependence of XMCD signal, the $\mathrm{MgO}$ capped Fe-NWs samples were subjected to $\mathrm{Ar}^{+}$ion sputtering.

\section{RESULTS AND DISCUSSIONS}

Typical FMR spectra for an array of Fe NWs at various field angles $\left(\theta_{h}\right)$ are presented in Fig. 1(b). Signal from the NWs can be reliably distinguished against the background at $\theta_{h}=0^{\circ}$, and its position can be traced as the angle $\theta_{h}$ deviates from $0^{\circ}$. The low-field background features, which are almost independent of $\theta_{h}$, originate from impurities inside the substrate and are excluded from further consideration. ${ }^{28,29}$ The angle dependences of the resonance field for $1 \mathrm{~A}$ and $2 \mathrm{~A}$ samples are shown in Fig. 2. Because the line widths of FMR spectra for these samples are broad, there is a finite uncertainty in determining the resonance field, which is shown as an error bar in the figure for each data point. The measurements were performed within 2 weeks of sample preparation. $H_{\text {res }}\left(\theta_{h}\right)$ dependence displays behavior expected for an oblate cylindrical ferromagnet characterized by shape anisotropy; that is, $H_{\text {res }}$ reaches a maximum when the magnetic field is directed along the short axis of the cylinder and diminishes as its direction deviates from this direction. ${ }^{30}$ However, the maximal and minimal values of $H_{r e s}$ are different for samples $1 \mathrm{~A}$ and $2 \mathrm{~A}$, which implies that they differ either in magnetization or in shape. It should be noted that

TABLE I. Width, thickness, and cap layer materials for the samples studied by FMR.

\begin{tabular}{|c|c|c|c|c|c|}
\hline Sample & $1 \mathrm{~A}$ & $1 \mathrm{~B}$ & & $2 \mathrm{~A}$ & $2 B$ \\
\hline Cap layer & \multicolumn{2}{|c|}{$\mathrm{Ag}(10 \mathrm{~nm})$} & & \multicolumn{2}{|c|}{$\operatorname{MgO}(20 \mathrm{~nm})$} \\
\hline NW width (nm) & & & 25 & & \\
\hline NW thickness (nm) & 4.5 & 7 & & 4.5 & 7 \\
\hline
\end{tabular}


(a)

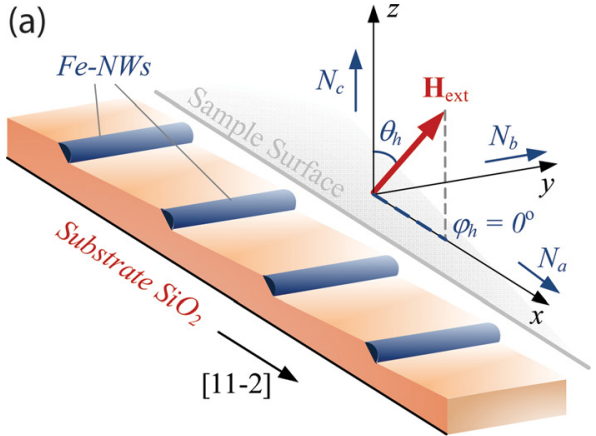

(b)

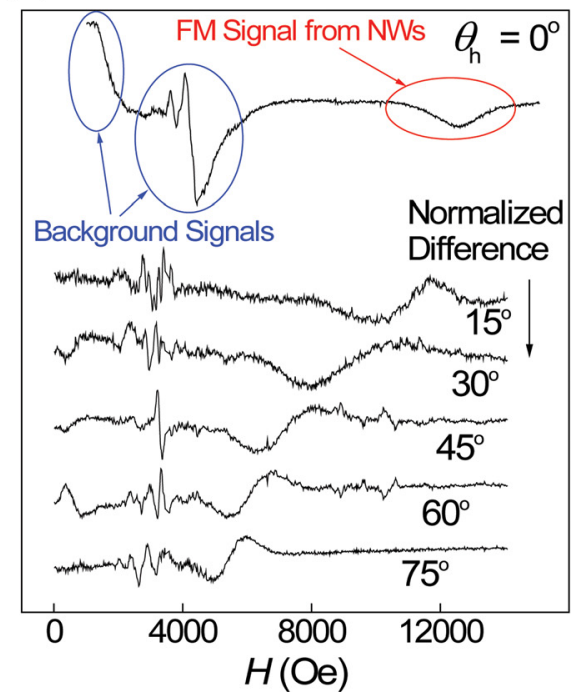

FIG. 1. (a) Schematic illustration of an array of NWs on a step-bunched oxidized vicinal Si (111) substrates and the experimental geometry used for FMR measurements. (b) Typical FMR spectra measured at $300 \mathrm{~K}$ for sample $1 \mathrm{~A}$ at various $\theta_{\mathrm{h}}$ values. Upper curve is the original FMR spectrum for $\theta_{\mathrm{h}}=0^{\circ}$. FMR spectra at other $\theta_{\mathrm{h}}$ values are normalized difference between the spectra with successive values of $\theta_{\mathrm{h}}$.

such a behavior is also characteristic of the $H_{\text {res }}\left(\theta_{h}\right)$ dependences for $1 \mathrm{~B}$ and $2 \mathrm{~B}$ samples (not shown). The difference between the angular dependence of $H_{R}$ for Set 1 and Set 2 are linked to the cap layer material and nature of the interface formed with the NWs. For MgO capped NW arrays (Set

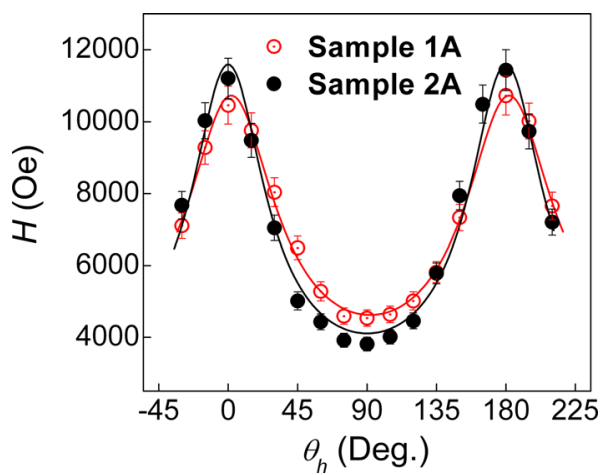

FIG. 2. Angular dependence of the resonance field measured at $300 \mathrm{~K}$ for the as-prepared samples $1 \mathrm{~A}$ and $2 \mathrm{~A}$. Open and solid circles show experimental data corresponding to samples $1 \mathrm{~A}$ and $2 \mathrm{~A}$, respectively. Solid lines curves are the angular dependencies calculated using Eq. (3). Error bar in obtaining the resonance field values for respective samples is also shown in the figure.
2), it is expected that an oxide interlayer would be formed between the $\mathrm{Fe} \mathrm{NWs}$ and $\mathrm{MgO}$, which reduces the effective magnetic volume (thickness of the NWs). Indeed, this is the case as will be shown later in the discussion.

In order to investigate the influence of the aging on the magnetic properties of the Fe-NW arrays, we present their FMR spectra at $300 \mathrm{~K}$ measured 2 and 5 months after the first measurement. Figure 3 shows the FMR spectra for both samples of Set 2 measured at different times. The effect of aging was also investigated for the Set 1 samples. We did not find any noticeable changes in the magnetic and resonance parameters with aging for the Set 1. From the angular dependence of the resonance fields for the samples of Set 2 (Fig. 3 ), we find that the resonance fields at $\theta_{h}=0^{\circ}$ and $180^{\circ}$ decrease after two months of aging, and most remarkably a difference appears between $H_{\text {res }}\left(0^{\circ}\right)$ and $H_{\text {res }}\left(180^{\circ}\right)$ for both samples. The latter fact, clearly visible in the sample $2 \mathrm{~A}$ and less noticeable in sample $2 \mathrm{~B}$, is a signature of a unidirectional anisotropy along the short axis of the nanowires. ${ }^{31}$ The magnitude of unidirectional anisotropy is linked to the thickness of the interfacial oxide layer which acts as a bias layer to pin the magnetization of the Fe-NWs. The thickness of this oxide interface layer for sample $2 \mathrm{~B}$ is not large enough to pin the whole volume of the Fe NWs. With further increase in aging time (five months) the strength of unidirectional anisotropy is decreased for sample $2 \mathrm{~A}$ and further enhanced for sample $2 \mathrm{~B}$. For the thinner sample $2 \mathrm{~A}$, the reduction in the strength of unidirectional anisotropy with
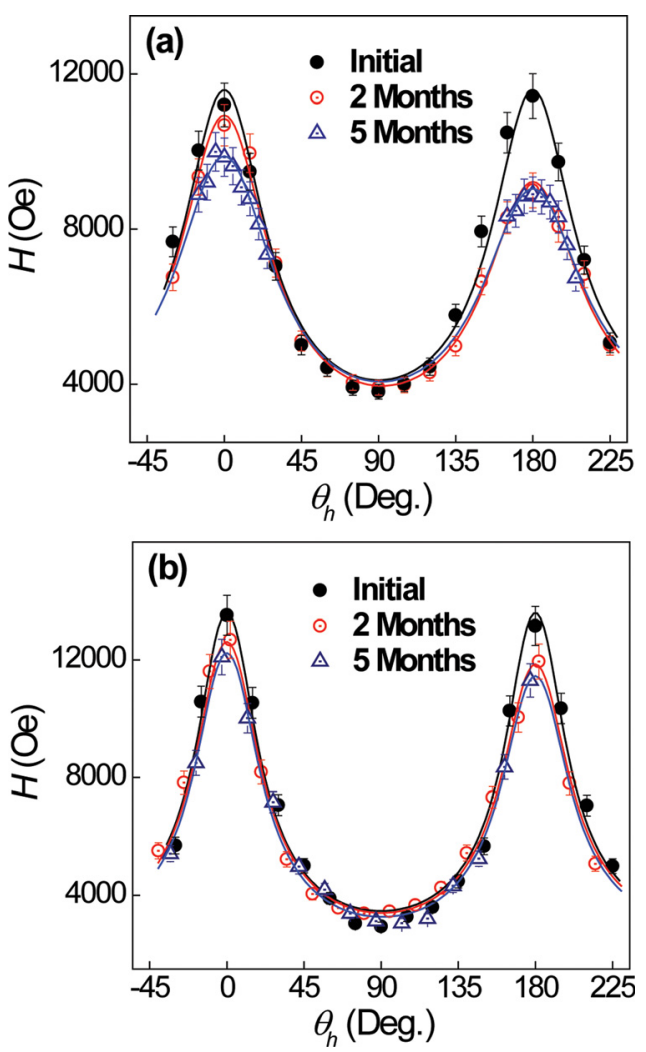

FIG. 3. Angular dependence of the resonance field measured at $300 \mathrm{~K}$ for (a) sample $2 \mathrm{~A}$ and (b) sample $2 \mathrm{~B}$ as a function of aging, that is, as prepared, and after 2 and 5 months aging under ambient conditions. Solid lines curves are the angular dependencies calculated using Eq. (3). Error bar in obtaining the resonance field values are also shown in the figure. 
extended aging is attributed to extensive oxidation of the NWs, resulting in a greatly reduced magnetization. A marginal increase in the strength of unidirectional anisotropy for sample $2 \mathrm{~B}$ for 5 month aged sample as opposed to the 2 months old sample suggests that the greater fraction of the NWs in the array have reduced thickness and contribute to enhancement of the unidirectional anisotropy strength. The thickness of the oxide interface layer is not enough to pin the whole volume of the NWs.

In order to obtain the magnetization parameters and understand the observed unidirectional anisotropy and its correlation with effective interface layer thickness (formed at the expense of FM volume), we analyze the FMR data using the Smit-Beljers ${ }^{30,32}$ approach. The resonance conditions for the uniformly magnetized ferromagnetic ellipsoid can be written in the form

$$
\begin{aligned}
\left(\frac{\omega}{\gamma}\right)^{2} & =\frac{1}{M^{2} \sin ^{2}(\theta)}\left[\frac{\partial^{2} U}{\partial \varphi^{2}} \frac{\partial^{2} U}{\partial \theta^{2}}-\left(\frac{\partial U}{\partial \theta \partial \varphi}\right)^{2}\right] ; \\
\frac{\partial U}{\partial \theta} & =0, \quad \frac{\partial U}{\partial \varphi}=0,
\end{aligned}
$$

where $U$ is the free energy, $\omega$ is the resonance frequency, $\gamma$ is the gyromagnetic ratio, and $M$ is the sample magnetization.

In spherical coordinates, $\mathbf{H}=\left(H \cos \varphi_{h} \sin \theta_{h}, H \sin \varphi_{h}\right.$ $\left.\sin \theta_{h}, \quad H \cos \theta_{h}\right)$ and $\quad \mathbf{M}=(M \cos \varphi \sin \theta, \quad M \sin \varphi \sin \theta$, $M \cos \theta)$. Thus, for our particular case, the expression for the free energy $U$ reads

$$
\begin{aligned}
U= & U_{Z}+U_{F}+U_{u d}=-M H\left[\sin \theta \sin \theta_{h} \cos \left(\varphi-\varphi_{h}\right)\right. \\
& \left.+\cos \theta \cos \theta_{h}\right]+\frac{1}{2} M^{2}\left(N_{a} \sin ^{2} \theta \cos ^{2} \varphi\right. \\
& \left.+N_{b} \sin ^{2} \theta \sin ^{2} \varphi+N_{c} \cos ^{2} \theta\right)+M H_{u d} \cos \theta
\end{aligned}
$$

where $U_{Z}$ is the Zeeman energy, $U_{F}$ is the demagnetizing energy for the ellipsoid with principal axes $a, b, c$, and demagnetizing factors $N_{a}, N_{b}$, and $N_{c}$, respectively. The last term, $U_{u d}$, is the unidirectional anisotropy energy due to the unidirectional anisotropy field $H_{u d .}{ }^{31}$

The characteristic feature of the NWs under investigation is the large ratio of the NW's length to width, which makes it possible to consider the shape of these objects as highly elongated ellipsoids with $b \gg a>c$ (see Fig. 1 for explanation of notations). This, in turn, means that $N_{b}=0$ and $N_{a}, N_{c} \neq 0$. Since $N_{a}+N_{b}+N_{c}=4 \pi$, we can write $N_{a}=4 \pi-N_{c}$. Having substituted the expression for the free energy (2) in a system (1), one obtains the system of equations for the calculation of the out-of-plane resonance field for the case where $\varphi_{H}=\varphi=0^{\circ}$

$$
\begin{aligned}
\left(\frac{\omega}{\gamma}\right)^{2}= & {\left[H_{\text {res }} \cos \left(\theta-\theta_{h}\right)+\left(4 \pi-2 N_{c}\right) M \cos (2 \theta)-H_{u d} \cos \theta\right] } \\
& \times\left[H_{r e s}\left(\sin \theta_{h} / \sin \theta\right)-\left(4 \pi-N_{c}\right) M\right] \\
H_{\text {res }}= & \frac{\left(4 \pi-2 N_{c}\right) M \sin (2 \theta)+2 H_{u d} \sin \theta}{2 \sin \left(\theta_{h}-\theta\right)} .
\end{aligned}
$$

We used Eq. (3) to fit the experimental $H_{\text {res }}\left(\theta_{h}\right)$ dependences for the samples under study and calculate the $M, N_{c}$, and $H_{u d}$ parameters for each of the samples. As seen from
Figs. 2 and 3, the fitted curves (solid lines) agree well with the experimental ones, which imply that the above approach describes quite well the behavior of the Fe NWs.

The analysis of the angle dependences of the resonance field for the sample $2 \mathrm{~A}$ shows that the effective magnetization of the sample diminishes from 990 to $790 \mathrm{emu} / \mathrm{cm}^{3}$ as a result of 5 months of aging. Along with the magnetization reduction, aging gives rise to the appearance of unidirectional anisotropy, which manifests itself in the difference between $H_{\text {res }}\left(0^{\circ}\right)$ and $H_{\text {res }}\left(180^{\circ}\right)$ (see Fig. 3). The calculated value of the unidirectional anisotropy field, $H_{u d}$, for the 2 and 5 months-aged sample 2A are 860 and $420 \mathrm{G}$, respectively. Evidence of aging is also present in sample $2 \mathrm{~B}$, in spite of the Fe NWs greater thickness. The effective magnetization, $M$, of sample 2B reduces from 1100 to $970 \mathrm{emu} / \mathrm{cm}^{3}$, and there appears a unidirectional anisotropy with $H_{u d} \approx 380 \mathrm{G}$ after 2-months-aging of the sample. The magnitude of the unidirectional anisotropy increases marginally to $400 \mathrm{G}$ after 5-months-aging. The results of the analysis are summarized in Table II.

Next, we examine the influence of aging on the demagnetization factors. One of the parameters of the system of equations (3) is the demagnetizing factor $N_{c}$ along the Fe NW thickness (see Fig. 1). Within the approximation $(b \gg a, c)$, one can easily express the ratio of the NW width $w$ to thickness $t$ as $^{33}$

$$
\frac{w}{t}=\frac{N_{c}}{4 \pi-N_{c}} .
$$

As a result of sample aging (oxidation), the $t$ and $w$ parameters can undergo some changes, and this change will give rise to changes in $N_{c}$. Assuming that the oxidation at the interface between $\mathrm{Fe}$ and $\mathrm{MgO}$ affects only the thickness of Fe NWs (i.e., the width $w$ of the NWs remains constant), one can calculate the ratio of the NW thicknesses before $\left(t_{1}\right)$ and after $\left(t_{2}\right)$ the sample aging

$$
\frac{t_{2}}{t_{1}}=\frac{4 \pi / N_{c 2}-1}{4 \pi / N_{c 1}-1},
$$

where $N_{c 1}$ and $N_{c 2}$ are the NW demagnetizing factors before and after the sample aging, respectively.

The samples of Set 1 and Set 2 were fabricated with the same nominal parameters $w$ and $t$ and under the same fabrication conditions. However, the FMR investigations show that the demagnetizing factor is larger for sample $2 \mathrm{~A}$ as compared with the sample $1 \mathrm{~A}\left(N_{c}^{\text {sample } 2 \mathrm{~A}}=9.5\right.$ and $\left.N_{c}^{\text {sample } 1 \mathrm{~A}}=9.1\right)$. This points to the conclusion that the $\mathrm{Fe}$

TABLE II. Magnetic parameters determined from the analysis of FMR data.

\begin{tabular}{lcccc}
\hline \hline Sample & Age (months) & $\mathrm{N}_{\mathrm{c}}$ & $\mathrm{M}_{\mathrm{eff}}$ (Gauss) & $\mathrm{H}_{\mathrm{ud}}$ (Gauss) \\
\hline 2A & 0 & 9.5 & 990 & $\ldots$ \\
& 2 & 9.3 & 840 & 860 \\
& 5 & 9.1 & 790 & 420 \\
2B & 0 & 10.3 & 1100 & $\ldots$ \\
& 2 & 10.2 & 970 & 380 \\
& 5 & 10.1 & 920 & 400 \\
\hline \hline
\end{tabular}


NW thickness is smaller in the sample $2 \mathrm{~A}$ as compared with the sample $1 \mathrm{~A}$. The same relation also holds for samples $2 \mathrm{~B}$ and 1B. Based on Eq. (5), one can calculate the difference between the thicknesses of the Fe NWs capped with $\mathrm{Ag}$ and $\mathrm{MgO}$. For both the thinner (group A) and thicker (group B) Fe NWs, the reduction in NW thickness as a consequence of the $\mathrm{MgO}$ capping is almost the same and is about $1 \mathrm{~nm}$ (as compared with the as-prepared samples).

It is interesting to analyze the effect of aging on the change in the demagnetizing factors for the $\mathrm{MgO}$ capped samples of Set 2. For the sample with the thicker Fe NWs (sample 2B), $N_{c}$ is almost constant with aging, which indicates that the reduction in NW thickness is small in comparison with the NW thickness. For the sample with the thinner Fe NWs (sample 2A), $N_{c}$ changed from 9.5 to 9.1 as a result of the 5 months-aging. This implies the reduction in the $w / t$ ratio, which suggests that the oxidation occurs not only across the NW thickness but also across the NW width.

From the analysis of the FMR data we infer that the FeNWs capped with $\mathrm{MgO}$ layer exhibit a reduction in effective volume of the NWs. All the results obtained can be reasonably explained assuming that the capping of the Fe NWs with $\mathrm{MgO}$ layer induces the oxidation processes at the interface between $\mathrm{Fe}$ and $\mathrm{MgO}$. The appearance of the nonstoichiometric iron oxide layer gives rise to reduction in the Fe NW thickness (as a rule) and appearance of the unidirectional anisotropy, where the latter reflects the exchange bias between the interfacial antiferromagnetic iron oxide and ferromagnetic $\mathrm{Fe}$ NWs. ${ }^{4}$ It appears that there are competing anisotropies within the system, namely, shape anisotropy and unidirectional anisotropy. This is further explained below.

The origin of observed out-of-plane unidirectional anisotropy in $\mathrm{MgO}$ capped $\mathrm{Fe}-\mathrm{NW}$ arrays can be understood from the finite size distribution of the step-bunched facets heights and terrace width. Such a distribution is a result of the self-assembled nature of the step bunched oxidized Si(111) templates. ${ }^{12,27}$ This induces variation in width and thickness of the wires within the array. It suggests that with the increased oxidation, effective thickness and width of the NWs having smaller thickness and width than the average thickness and width of the NW array would reach a regime that leads to a spin reorientation transition. As aging progresses, the proportion of wires exhibiting this behaviour increases leading to an increasing prevalence of perpendicularly-coupled regions. In previous studies, it has been shown that $\mathrm{Fe}$ does exhibit a spin reorientation transition with Fe-thickness in the range of 4-8 monolayers. ${ }^{34,35} \mathrm{It}$ is well known that the materials with perpendicular easy axis and in contact with a hard layer (AFM) exhibits an out-of plane unidirectional anisotropy. ${ }^{36}$ Our case is very similar to this, with Fe-NWs of reduced thickness exhibiting spinreorientation transition and proximity of the $\mathrm{FeO}$ interface layer facilitates the observation of the out-of-plane unidirectional anisotropy. This anisotropy competes with the shape anisotropy, which is still the dominant one even after 5 months of aging. The above model also explains the shallower dependence of this unidirectional anisotropy in the thicker NW array (2B). In a recent report, Fan et al. ${ }^{37}$ showed that the $\mathrm{Fe}-\mathrm{MgO}$ interface possess a non-collinear spin structure leading to the observation of unidirectional anisotropy in the interfacial region. They reported its strength to be strongly dependent on the oxygen defects and disorder in the interfacial region. Observation of unidirectional anisotropy in our case at $300 \mathrm{~K}$ is a curious observation considering the fact that the Neel temperature of $\mathrm{Fe}-\mathrm{O}$ phase is $\sim 180 \mathrm{~K}$. This suggests that the Fe-O formed at the $\mathrm{NW}-\mathrm{MgO}$ interfaces is likely to possess large concentration of defects and disorder owing to the polycrystalline nature of the wires. It leads to an enhanced Neel temperature as the $\mathrm{Fe}-\mathrm{O}$ phase shows an increase in $\mathrm{T}_{\mathrm{N}}$ with increasing defect concentration. ${ }^{38}$

In order to support our model, we report XAS and XMCD spectra measured on a Fe-NW array sample with similar properties as sample $2 \mathrm{~A}(10 \mathrm{~nm} \mathrm{MgO}$ capped with $\sim 4 \mathrm{~nm}$ Fe thickness) but measured after three weeks and six weeks of sample preparation. Fig. 4 shows the respective XAS spectra for this sample measured at $300 \mathrm{~K}$ with positive and negative helicity. The corresponding XMCD spectra are shown in the bottom panels of the figure. The spectra were normalized to the incident flux and pre-edge intensity. The XAS line shape shows clear signature of oxidation
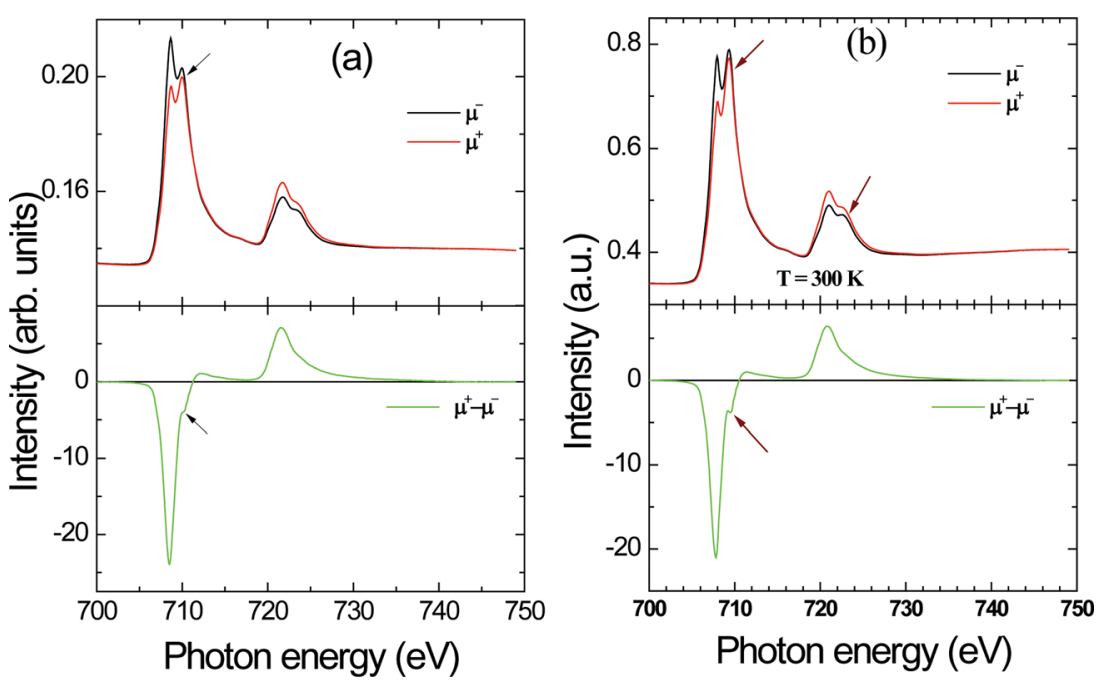

FIG. 4. X-ray absorption spectra (upper panels) and corresponding XMCD $\left(\mu^{+}-\mu^{-}\right)$spectra (lower panels) measured at 300 for the $\mathrm{Fe} L_{3,2}$ edge for a Fe NW array sample with similar wire dimensions to sample $2 \mathrm{~A}$ and capped with $10 \mathrm{~nm} \mathrm{MgO}$ after (a) 3 weeks and (b) 6 weeks of deposition. The spectra were recorded in the TEY mode with an incidence angle of $70^{\circ}$ relative to the surface normal for magnetization parallel and antiparallel to the x-ray helicity (black and red curves). The arrow indicates the absorption peak and magnetic circular dichroism (MCD) related to oxidized Fe. 
compared to the metallic Fe (Ref. 39) owing to the presence of oxidized interface with the cap layer of $\mathrm{MgO}$ and hybridization of $\mathrm{O}_{2 \mathrm{p}}-\mathrm{Fe}_{3 \mathrm{~d}}$ states. From the XAS spectra (upper panel), we notice that the oxide peak at $1.2 \mathrm{eV}$ above the $\mathrm{Fe}$ $\mathrm{L}_{3}$ edge is present in both cases. The oxide peak (marked with arrow) could originate from various phases of iron oxide that could form due to oxidation of the interfaces with the $\mathrm{MgO}$ cap layer. The magnitude of this oxide peak is found to increase with aging, suggesting that the thickness of the interfacial oxide layer has increased with aging due to the reaction between the $\mathrm{Fe}$ and $\mathrm{MgO}$. The XMCD signal determined from the XAS spectra are also shown in the lower panels. Here, $\mu^{+}\left(\mu^{-}\right)$refers to the absorption coefficient for the photon helicity parallel (antiparallel) to the $\mathrm{Fe}$ $3 \mathrm{~d}$ majority spin direction. The XMCD sum rules ${ }^{40,41}$ were applied to estimate the spin magnetic moments of the Fe. The estimated values of $\mathrm{Fe}$ spin moment for 3 and 6 weeks aged samples at $300 \mathrm{~K}$ are found to be 1.95 and $1.80 \mu_{\mathrm{B}} /$ atom. For the estimate of spin $\left(\mathrm{m}_{\mathrm{s}}\right)$ and orbital $\left(\mathrm{m}_{\mathrm{l}}\right)$ moment contributions, we assumed $n_{h}$ (number of holes in the $3 d$ states) to be 3.4 for iron ${ }^{40}$ and a negligible value of the spin dipolar term. From the XMCD spectra one can clearly see that presence of oxide induces a reduction of the magnetic moment per $\mathrm{Fe}$ atom, due to the formation of $\mathrm{Fe}-\mathrm{O}$.

In order to check the oxide formed at the $\mathrm{SiO}_{2}-\mathrm{Fe} \mathrm{NW}$ interface, we etched the $\mathrm{MgO}$ cap layer of a thick Fe-NW sample $(7 \mathrm{~nm}$ Fe thickness and $10 \mathrm{~nm} \mathrm{MgO}$ thickness) using the $\mathrm{Ar}^{+}$ion etching and studied its XAS as a function of etch depth. As shown in Fig. 5, after the first etch $\left(\mathrm{Ar}^{+}\right.$ions etching for $15 \mathrm{~min}$ that corresponds to the etch thickness comparable to $\mathrm{MgO}$ cap layer thickness), XAS spectra show a much reduced signal corresponding to hybridization of oxygen with Fe. In addition to the reduction in oxide peak intensity, we also notice that the effective magnetic moment of $\mathrm{Fe}$ is further reduced by $15 \%$ (corresponding XMCD spectra are not shown). This is possibly related to the ion damage. With a further $\mathrm{Ar}^{+}$ion etch step to reach the $\mathrm{Fe}-\mathrm{SiO}_{2}$ interface, we find that after second etching thickness of the Fe-NWs is significantly reduced $(\sim 1 \mathrm{~nm}, \sim$ escape depth of electrons) we did not observe any enhancement in oxide signal arising

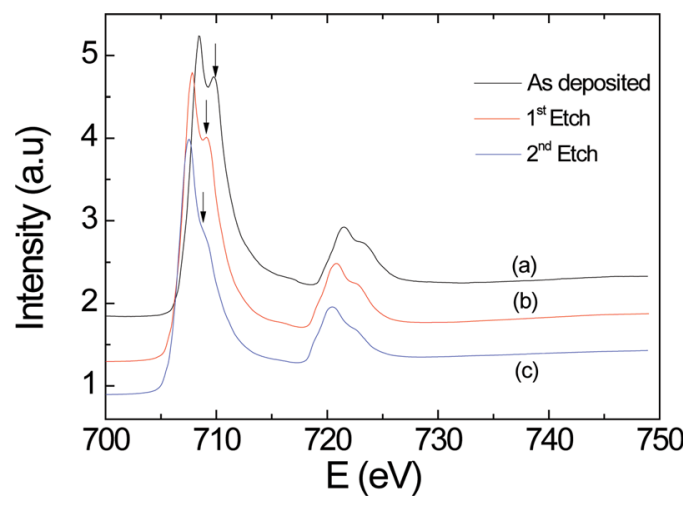

FIG. 5. The XAS spectra of a Fe NW array sample with positive helicity measured in the TEY mode at an incidence angle of $70^{\circ}$ and recorded at $300 \mathrm{~K}$ (a) after 3 weeks of deposition, and after two $\mathrm{Ar}^{+}$ion etching cycles (curve $\mathrm{b}$ and $\mathrm{c}$ ), leading to removal of $\mathrm{MgO}$ and oxidized $\mathrm{Fe}$. The arrow indicates the absorption peak related to oxidized Fe.

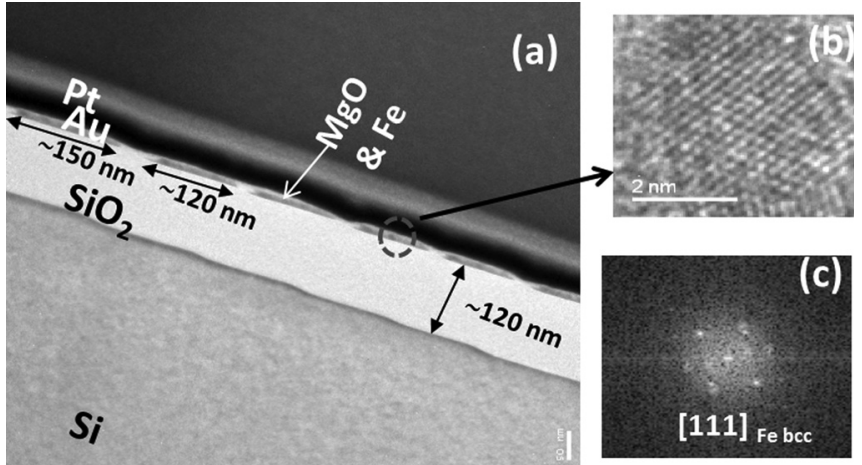

FIG. 6. (a) Cross-sectional TEM image of a $5 \mathrm{~nm}$ thick Fe-NW array on a $110 \mathrm{~nm}$ average periodicity oxidized Si template grown under similar deposition conditions to Set 1 and Set 2, but with deposition flux directed towards the descending step (downhill) direction. The average wire width of the NWs varies between 90 and $120 \mathrm{~nm}$. (b) High-resolution image showing the lattice of a Fe-NW crystallite. (c) Corresponding Fast Fourier Transform (FFT) of the region shown in (b) showing the Fe bcc-crystal structure projected on its [111] zone axis.

from the $\mathrm{Fe}-\mathrm{SiO}_{2}$ interface. Only a weak oxide peak in the form of a small hump is present (curve $\mathrm{c}$ in Fig. 5). This suggests that the reaction rate of the $\mathrm{Fe}$ with $\mathrm{SiO}_{2}$ is significantly smaller as compared with $\mathrm{MgO}$. This is also evident from the cross-section TEM studies performed on a $\mathrm{MgO}$ capped Fe-NW array within 6 weeks of deposition which showed that an oxide interlayer is formed between the Fe NWs and $\mathrm{MgO}$ cap layer due to oxidation reaction of $\mathrm{Fe}$ with $\mathrm{MgO}$ (Fig. 6). From the TEM analysis we infer that the $F e$-NWs possess polycrystalline nature, and its crystal structure is $b c c$ (Figs. 6(b) and 6(c)). The structure of the oxide layer crystal structure is $f c c$ and resembles the $\mathrm{FeO}$ structure (fcc with rock-salt symmetry). This further corroborates our model that the presence of $\mathrm{MgO}$ cap layer facilitates formation of the oxide interlayer.

Fig. 7 shows the magnetic hysteresis loop (HL) determined from the XMCD spectra measured after six weeks of deposition at $300 \mathrm{~K}$ for the $\mathrm{Fe}-\mathrm{L}_{3}$ edge with $\mathrm{x}$-rays incident at an angle of 0 and $70^{\circ}\left(0^{\circ}\right.$ and $70^{\circ}$ loops corresponds to out-of plane and in-plane magnetic field directions) and

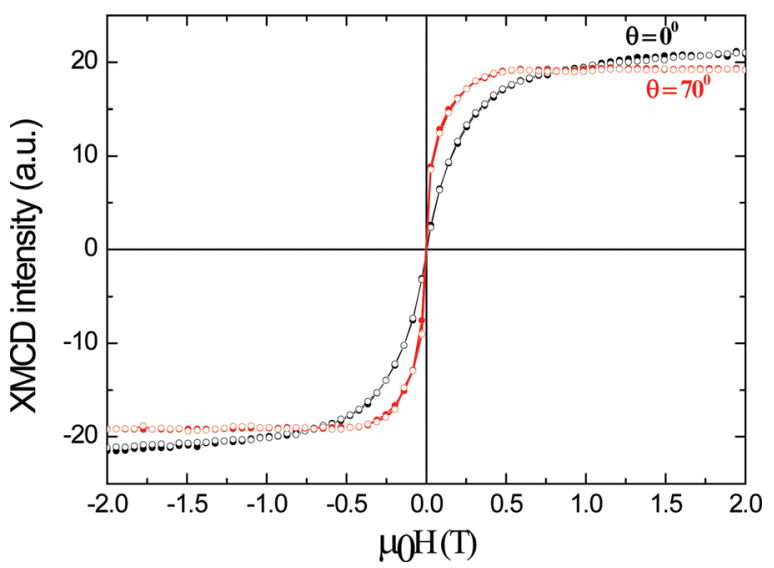

FIG. 7. The XMCD hysteresis loops measured at $300 \mathrm{~K}$ with $\mathrm{X}$-rays incident at an angle of $0^{\circ}$ and $70^{\circ}\left(0^{\circ}\right.$ and $70^{\circ}$ loops correspond to out-of plane and in-plane magnetic field directions) for a Fe-NW array sample. Spin moments are derived from the asymmetry found between the $\mathrm{Fe}-\mathrm{L}_{3}$ edge for positive and negative helicity using sum rules. 
measured at $300 \mathrm{~K}$ for the second sample. The analysis suggests that the in-plane uniaxial anisotropy is the dominant anisotropy. This further supports our suggestion that the out of plane unidirectional component observed in Fe-NWs with aging is related to the formation of an oxide interface and areas of reduced thickness within the Fe NW arrays that facilitates stabilization of the out-of-plane unidirectional anisotropy component. The magnitude of this anisotropy increases with the aging as more and more regions within the NW arrays with reduced thickness are formed leading to an enhanced out of plane unidirectional component.

\section{CONCLUSIONS}

In summary, with the use of FMR technique, we studied the effects of aging on the magnetic parameters of the thin Fe NWs arrays. Aging did not result in any noticeable changes of the parameters of the samples of the Set 1 (Ag-capped Fe NW arrays) but strongly affected the magnetic parameters of the samples of the Set 2 (MgO capped NW arrays). For the latter, the effective magnetization reduced by about $200 \mathrm{emu} / \mathrm{cm}^{3}$, and there appeared changes in demagnetizing factors, with both of these effects pointing to the fact that the presence of the $\mathrm{MgO}$ layer induced (accelerated) oxidation processes at the interface between a $\mathrm{Fe}$ $\mathrm{NW}$ and $\mathrm{MgO}$ cap layer. At the same time, the unidirectional anisotropy along the normal to the sample plane was revealed in the samples of group 2. This phenomenon is strongly expressed in the sample with the Fe NWs nominal thickness $t=4.5 \mathrm{~nm}$ (the difference between $H_{\text {res }}\left(0^{\circ}\right)$ and $H_{\text {res }}\left(180^{\circ}\right)$ is $\left.\Delta H_{\text {res }} \approx 1500 \mathrm{Oe}\right)$ and less pronounced in the sample with $t=7 \mathrm{~nm}$ (the above difference is near $400 \mathrm{Oe}$ ). Our results highlight some of the unusual features of unidirectional anisotropy in ferromagnetic-oxide interfaces in magnetic nanostructures. These results are relevant to other magnetic nano-structures in which the elemental constituents are likely to form an antiferromagnetic oxide (for example, $\mathrm{FeO}, \mathrm{CoO}, \mathrm{MnO}, \mathrm{NiO}$ ), as is the case of numerous magnetic or multiferroic oxide heterostructures.

\section{ACKNOWLEDGMENTS}

Work supported by the Science Foundation Ireland (SFI) under Contract No. 06/IN.1/I91, ERA-Net NANOWAVE program, the Irish Government's Program (ISPIRE) for Research in Third Level Institutions, Cycle 4, National Development Plan 2007-2013, the Spanish Ministerio de Ciencia y Innovación (EUI2008-03884 and PTA2008-1108-I), Agència de Gestió d'Ajuts Universitaris i de Recerca (2009 SGR 695), and Nanoaracat.

${ }^{1}$ J. Nogues, J. Sort, V. Langlais, V. Skumryev, S. Surinach, J. S. Munoz, and M. D. Baro, Phys. Rep. 422, 65-117 (2005).

${ }^{2}$ A. Hoffmann, M. Grimsditch, J. E. Pearson, J. Nogues, W. W. A. Macedo, and I. K. Schuller, Phys. Rev. B 67, 220406 (2003).

${ }^{3}$ F. Radu and H. Zabel, "Exchange bias effect of ferro/antiferromagnetic heterostructures," Springer Tracts Mod. Phys. 227, 97-184 (2008).
${ }^{4}$ R. L. Stamps, J. Phys. D 33, R247 (2000).

${ }^{5}$ T. Mewes, H. Nembach, J. Fassbender, H. Hillebrands, J.-V. Kim, and R. L. Stamps, Phys. Rev. B 67, 104422 (2003).

${ }^{6}$ R. Abrudan et al. Phys. Rev. B 77, 014411 (2008).

${ }^{7}$ M. Gruyters and D. Schmitz, Phys. Rev. Lett. 100, 077205 (2008).

${ }^{8}$ S. A. Wolf, D. D. Awschalom, R. A. Buhrman, J. M. Daughton, S. Von Molnar, M. L. Roukes, A. Y. Chtchelkanova, and D. M. Treger, Science 294, 1488 (2001).

${ }^{9}$ F. Radu, R. Abrudan, I. Radu, D. Schmitz, and H. Zabel, Nat. Commun. 3, 1728 (2011).

${ }^{10}$ J. H. Jung, S. H. Lim, and S. R. Lee, Appl. Phys. Lett. 101, 242403 (2012).

${ }^{11}$ S. Matt, K. Takano, S. S. P. Parkin, and E. E. Fullerton, Phys. Rev. Lett. 87, 087202 (2001).

${ }^{12}$ S. K. Arora, B. J. O’Dowd, B. Ballesteros, P. Gambardella, and I. V. Shvets, Nanotechnology 23, 235702 (2012).

${ }^{13}$ H. Zheng, R. Skomski, L. Menon, Y. Liu, S. Bandyopadhyay, and D. J. Sellmyer, Phys. Rev. B 65, 134426 (2002).

${ }^{14}$ K. R. Pirota, E. L. Silva, D. Zanchet, D. Navas, M. Vazquez, M. Hernandez-Velez, and M. Knobel, Phys. Rev. B 76, 233410 (2007).

${ }^{15}$ Y. Otani, A. Nemoto, S. G. Kim, K. Fukamichi, O. Kitakami, and Y. Shimada, J. Magn. Magn. Mater. 198-199, 434 (1999).

${ }^{16}$ A. Nemoto, Y. Otani, S. G. Kim, K. Fukamichi, O. Kitakami, and Y. Shimada, Appl. Phys. Lett. 74, 4026 (1999).

${ }^{17}$ J. Sort, M. Fraune, C. Koenig, B. Beschoten, and G. Guntherodt, Appl. Phys. Lett. 84, 3696 (2004).

${ }^{18}$ M. Fraune, U. Rudiger, G. Guntherodt, C. Cardoso, and P. Freitas, Appl. Phys. Lett. 77, 3815 (2000).

${ }^{19}$ S. Mao, J. Giusti, N. Amin, J. van Ek, and E. Murdock, J. Appl. Phys. 85, 6112 (1999).

${ }^{20}$ Y. Shen, Y. Wu, H. Xie, K. Li, J. Qiu, and Z. Guo, J. Appl. Phys. 91, 8001 (2002).

${ }^{21}$ S. H. Chung, A. Hoffmann, and M. Grimsditch, Phys. Rev. B 71, 214430 (2005).

${ }^{22}$ H. Oh, S. B. Lee, J. Seo, H. G. Min, and J. S. Kim, Appl. Phys. Lett. 82, 361-363 (2003).

${ }^{23}$ X. G. Zhang, W. H. Butler, and A. Bandyopadhyay, Phys. Rev. B 68, 092402 (2003).

${ }^{24}$ C. Tusche, H. L. Meyerheim, N. Jedrecy, G. Renaud, and J. Kirschner, Phys. Rev. B 74, 195422 (2006).

${ }^{25}$ M. Bibes, J. E. Villegas, and A. Barthelemy, Adv. Phys. 60, 5-84 (2011).

${ }^{26}$ C. H. Lambert, A. Rajanikanth, T. Hauet, S. Mangin, E. E. Fullerton, and S. Andrieu, Appl. Phys. Lett. 102, 122410 (2013).

${ }^{27}$ S. K. Arora, B. J. O'Dowd, P. C. McElligot, I. V. Shvets, P. Thakur, and N. B. Brookes, J. Appl. Phys. 109, 07B106 (2011).

${ }^{28}$ M. D. Glinchuk, I. P. Bykov, S. M. Kornienko et al., J. Mater. Chem. 10, 941 (2000).

${ }^{29}$ A. I. Tovstolytkin, V. V. Dzyublyuk, D. I. Podyalovskii, X. Moya, C. Israel, D. Sánchez, M. E. Vickers, and N. D. Mathur, Phys. Rev. B 83, 184404 (2011).

${ }^{30}$ A. G. Gurevich and G. A. Melkov, Magnetization Oscillations and Waves (CRC Press, Boca Raton, 1996).

${ }^{31}$ S. Chikazumi, Physics of Ferromagnetism (Oxford University Press, New York, 2005).

${ }^{32}$ J. Smit and H. G. Beljers, Phillips Res. Rep. 10, 113 (1955).

${ }^{33}$ J. A. Osborn, Phys. Rev. 67, 351 (1945).

${ }^{34}$ M.-T. Lin, J. Shen, W. Kuch, H. Jenniches, M. Klaua, C. M. Schneider, and J. Kirschner, Phys. Rev. B 55, 5886 (1997).

${ }^{35}$ A. Kukunin, J. Prokop, and H. J. Elmers, Phys. Rev. B 76, 134414 (2007).

${ }^{36}$ K. Yakushiji, T. Saruya, H. Kubota, A. Fukushima, T. Nagahama, S. Yuasa, and K. Ando, Appl. Phys. Let. 97, 232508 (2010).

${ }^{37}$ Y. Fan, K. J. Smith, G. Lupke, A. T. Hanbicki, R. Goswami, C. H. Li, H. B. Zhao, and B. T. Jonker, Nat. Nanotechnol. 8, 438 (2013).

${ }^{38}$ F. B. Koch and M. E. Fine, J. Appl. Phys. 38, 1470 (1967).

${ }^{39}$ T. J. Regan, H. Ohldag, C. Stamm, F. Nolting, J. Luning, J. Stohr, and R. L. White, Phys. Rev. B 64, 214422 (2001).

${ }^{40}$ C. T. Chen, Y. U. Idzerda, H. J. Lin, N. V. Smith, G. Meigs, E. Chaban, G. H. Ho, E. Pellegrin, and F. Sette, Phys. Rev. Lett. 75, 152-155 (1995).

${ }^{41}$ P. Carra, B. T. Thole, M. Altarelli, and X. Wang, Phys. Rev. Lett. 70, 694 (1993). 